\title{
Patient Safety and Patients' Rights
}

\author{
Ross D. Silverman, JD, MPH \\ Virtual Mentor. June 2004, Volume 6, Number 6.
}

Covering the Uninsured.

Adopting a uniform system that enhances patient safety.

Upon initial examination, efforts toward achieving these 2 goals appear philosophically complementary. For example, both tout as a primary benefit improvement of access to health care services. However, closer inspection of these initiatives' more prominent features reveals seemingly irreconcilable demands. As described below, the push toward consumer-driven health plans as a way to offer affordable employer-based health coverage (and, hence, advance the first goal) requires that comprehensive information be available about individual physicians. The patient safety movement's advocacy for the creation of a confidential medical error reporting system (that would serve the second goal) requires safeguarding individual physician information from the public.

\section{Consumer-Driven Health Care}

With more than 43 million Americans lacking health insurance and health insurance premiums for the insured rising by double digits year after year, we appear to be approaching the demise of the current structure of the employment-based health insurance system [1]. Employers, seeking both new ways to offer employees the valuable benefit of health insurance coverage and, at the same time, better ways to contain the financial risk they shoulder when providing such benefits, are increasingly turning to consumer-driven health benefit plans as a possible solution [2-4]. Recently approved by the Internal Revenue Service, consumer-driven (or consumer-directed) health plans continue the economic trend toward turning passive patients who may overutilize health services into savvy, frugal consumers of health services [5-7] by "putting patients in a position to say 'no' to themselves" [4]. While there are several different ways to structure a "consumer-driven" health plan [2-4], they generally offer individual employees an increased role in the design of their benefit package, including choice of physician and the opportunity to set the level of their financial risk. In a consumer-directed plan, patients shoulder a greater burden of the cost of health services.

In addition to making cost-benefit analyses of particular interventions, individual patients will have a greater responsibility to research and analyze the quality of their clinicians and to weigh the relative merits of these providers' expertise in the particular interventions. There are grave concerns that patients are being moved into these plans with neither the requisite training to expertly assess quality data, nor even an adequate level of available data from which to base quality comparisons. Meanwhile, hundreds of thousands, if not millions, of consumers are already joining such plans, and they will need access to sophisticated data on the quality of 
health care professionals to make informed decisions about how best to expend their health care dollars.

\section{Confidential Error and Near-Miss Reporting}

As the medical malpractice insurance/tort reform debate rages on in Congress and in state legislatures nationwide, a concomitant discussion continues about ways to improve the safety of the system in which health care is delivered. Inspired in part by the seminal Institute of Medicine (IOM) Report To Err Is Human [8], the patient safety movement represents a paradigm shift away from the traditional models for both measuring medical quality and addressing circumstances in which less-than-ideal outcomes occur. Among the central features of the patient safety philosophy is the turn toward seeking improvements at the systems level rather than on singling out the clinician during whose watch the error or adverse outcome occurred. According to the IOM, "Discovering and fixing latent failures and decreasing their duration are likely to have a greater effect on building safer systems than efforts to minimize active errors at the point at which they occur" [9]. This focus on systems rather than on individuals is accompanied by a focus on prevention rather than on punishment. These values encourage health care professionals to participate in creating a culture of patient safety by helping to move the health care system away from the inefficient and often inaccurate "blame and shame" heaped upon individuals toward a more holistic view of quality improvement.

A successful patient safety system would also encourage the collection of detail-rich information about medical practice patterns, the types of errors which occur in both inpatient and outpatient settings, and rigorous examination of "near misses," those situations in which injury-free errors occur or injuries are narrowly averted. The most effective source of such information is believed to be the health care professionals working in the system. As evidenced by discussions surrounding the tort reform debate, however, fear of discovery, lawsuits, and bad publicity significantly decreases willingness to file reports and hinders the ability of the organization to create solutions for error-prone systems, practices, or practitioners. Furthermore, those willing to report safety concerns under the current system may face retaliation, alienation, and litigation by their peers or the institutions about whom they are reporting [10]. Consequently, patient safety advocates support the development of reporting systems with nonpunitive means of improving behavior, immunity for those willing to report errors, robust protection of confidentiality of the participants in the report, and protection against discovery via the litigation process [8].

The reporting system most prominently promoted as a model for patient safety is the Aviation Safety Reporting System run by NASA and the Federal Aviation Administration, which has received more than 500,000 safety reports since its introduction in 1990 and currently receives more than 2900 reports per month without a single breach of confidence of the reporter [11]. While such systems have their skeptics [12], the allure of improving patient care through a blame-free health delivery system has led to the development of a patchwork quilt of reporting systems based upon these principles. A lack of standardization among these systems has significantly hindered their usefulness in developing generalizable knowledge about the nature of medical errors or data upon which to established evidence-based medical practices [13]. However, the American Medical Association, Joint Commission on Accreditation of Healthcare Organizations and others support the creation of a confidential, voluntary national patient safety 
reporting system, and legislation in support of such a system has made significant progress through Congress [14].

\section{Conclusion}

Should a voluntary national patient safety reporting system be implemented and robustly supported by providers and Congress, the movement to gather patient safety data will result in information that can make dramatic, universal improvements in how the US health system delivers care and may lead to true standardization of medical practice nationwide. At that point, measurements of quality could theoretically be removed as a factor of competition in the health care marketplace. But until that point, patients who are increasingly more responsible for balancing quality and cost concerns when making health care service and physician choices must not be placed in a quality-of-care information vacuum. A balance must be achieved between fostering the collection of patient safety data and protecting the rights of patients to make informed, autonomous medical decision 


\section{References}

1. Enthoven AC. Employment-based health insurance is failing: now what? Health Aff. 2003;Suppl:W3-237-49.

2. Andrews M. Does it pay to manage your own care? New York Times. January 18, 2004:7.

3. Samuel TW, Raleigh SG, Hower JM, Schwartz RW. The next stage in the health care economy: aligning the interests of patients, providers, and third-party payers through consumer-driven health care plans. Am J Surg. 2003;186:117-24.

4. Gabel JR, LoSasso AT, Rice T. Consumer-driven health plans: are they more than talk now? Health Aff. 2002; W395-W407.

5. Mariner WK. Slouching toward managed care liability: reflections on doctrinal boundaries, paradigm shifts, and incremental reform. J Law Med Ethics. 2001; 29:253-277.

6. Jacobi JV. After managed care: gray boxes, tiers and consumerism. St. Louis L. J. 2003;47:397410.

7. Jacob JA. A consumer-driven future: when patients call the shots. Am. Med. News. Sept 23-30, 2002. Accessed October 2, 2003.

8. Committee on Quality of Health Care in America, Institute of Medicine, To Err Is Human: Building a Safer Health System. Washington DC: National Academy Press; 2000.

9. To Err Is Human, 66.

10. Twedt S. How the tables turn on doctors. Pittsburgh Post-Gazette. October 26. 2003: A1.

11. The Aviation Safety Reporting System. Accessed October 2, 2003.

12. Andrus $\mathrm{CH}$, Villasenor EG, Kettelle JB, Roth R, Sweeney AM, Matolo NM. "To Err Is Human": uniformly reporting medical errors and near misses, a naive, costly, and misdirected goal. $J$ Am Coll Surg. 2003;196:911-8.

13. Board on Health Care Services, Institute of Medicine, Patient Safety: Achieving a New Standard for Care. Washington DC: National Academy Press; 2004:247-316.

14. Palmisano DJ. Patient safety legislation-an urgent call to action. Am Med News, May 17, 2004. Accessed May 20, 2004. 